\title{
A COMPOSITE COINCIDENCE DEGREE WITH APPLICATIONS TO BOUNDARY VALUE PROBLEMS OF NEUTRAL EQUATIONS
}

\author{
L. H. ERBE, W. KRAWCEWICZ AND J. H. WU
}

\begin{abstract}
We present a topological degree theory for the nonlinear problem $L(I-B)(x)=G(x)$ with applications to a class of boundary value problems of neutral equations, where $L$ is an unbounded Fredholm operator of index zero, $B$ is condensing and $G$ is $L$-compact.
\end{abstract}

\section{INTRODUCTION}

The main purpose of this paper is to develop topological tools for the study of a nonlinear problem of the type

$$
L(I-B)(x)=G(x), \quad x \in \Omega,
$$

where $\mathbb{E}$ and $\mathbb{F}$ are Banach spaces, $\Omega \subseteq \mathbb{E}$ is an open and bounded subset, $L: \operatorname{Dom}(L) \subseteq \mathbb{E} \rightarrow \mathbb{F}$ is a Fredholm operator of index zero, $B: \bar{\Omega} \rightarrow \mathbb{E}$ is a condensing mapping and $G: \bar{\Omega} \rightarrow \mathbb{F}$ is an $L$-compact mapping.

The motivation for the study of such a nonlinear problem arises from boundary value problems of neutral functional differential equations. For example, consider a neutral equation of the form

$$
\frac{d}{d t}\left[x(t)-b\left(t, x_{t}\right)\right]=f\left(t, x_{t}\right)
$$

where $f: \mathbb{R} \times C\left([-r, 0] ; \mathbb{R}^{n}\right) \rightarrow \mathbb{R}^{n}$ is a completely continuous mapping, $b: \mathbb{R} \times$ $C\left([-r, 0] ; \mathbb{R}^{n}\right) \rightarrow \mathbb{R}^{n}$ is continuous and satisfies the following Lipschitz condition

$$
|b(t, \varphi)-b(t, \psi)| \leq k \cdot \sup _{-r \leq s \leq 0}|\varphi(s)-\psi(s)|,
$$

where $\varphi, \psi \in C\left([-r, 0] ; \mathbb{R}^{n}\right) ; k<1$ is a constant and $x_{t}(s)=x(t+s)$. We can show that, after certain manipulations, a general boundary value problem of

Received by the editors March 27, 1990.

1980 Mathematics Subject Classification (1985 Revision). Primary 34K10, 34K15, 47H09, 47H10, 47H15, 47H17.

Key words and phrases. Essential map, topological degree, topological transversality, coincidence problem, condensing map, boundary value problems, neutral equations, periodic solution.

The first two authors' research partially supported by NSERC-Canada.

The last author's research partially supported by J. Gordin Kaplan Award Postdoctoral Fellowship at the university of Alberta. 
a neutral equation (1.2) can be formulated as an abstract nonlinear functional equation of the form (1.1).

It is natural to expect the boundary value problem of the neutral equation (1.2) can be reduced to a corresponding problem of the retarded equation

$$
\dot{x}(t)=f\left(t, x_{t}\right)
$$

through the following deformation

$$
\frac{d}{d t}\left[x(t)-\lambda b\left(t, x_{t}\right)\right]=f\left(t, x_{t}\right), \quad \lambda \in[0,1] .
$$

Since boundary value problems of retarded equations have been extensively studied (see, e.g., [1, 3, 4, 9, 10, 15, 27, 29]), the importance of the above reduction is obvious. In the case where $b(t, \varphi)$ is linear in $\varphi$, this reduction for periodic boundary value problems has been done in [5], by developing a homotopy invariance of Mawhin's coincidence degree allowing a continuous deformation of linear parts (unbounded Fredholm operators). However, this method cannot be applied to the general situation where $b(t, \varphi)$ may be nonlinear in $\varphi$.

In this paper, we extend the notion of essential maps, introduced by Granas (cf. [8]) and we generalize the topological transversality theorem to the nonlinear problem (1.1). We also develop a topological degree theory to detect the essential maps. It is shown that our topological degree, called composite coincidence degree, includes Brouwer degree [2], Leray-Schauder degree [17], Nussbaum degree (cf. [23, 24]), Sadovskii degree [26], Hetzer degree [13], as special cases, and that the homotopy invariance property of this topological degree allows the aforementioned reduction under the existence of a priori bounds for solutions of the family of equations (1.5). Therefore, our degree theory provides a unified approach to allow one to extend various existence results of boundary value problems from retarded equations to neutral equations. In a further paper, we will apply our degree to investigate the multiplicity and bifurcation of the nonlinear problem (1.1) in the case where the a priori bounds are absent.

The rest of this paper is organized as follows. In the next section we introduce the concept of essential mappings, prove a topological transversality theorem and develop a topological degree theory for the nonlinear problem (1.1). The properties of the degree are formulated in $\S 3$. Section 3 contains also an example of periodic boundary value problems of neutral equations to illustrate our general theory. More general boundary value problems are discussed in $\S 4$ by using the continuation property of our degree and an extension of the guiding function method.

\section{CoMposite COINCIDENCE DEGREE}

Let us recall the notion of an abstract measure of noncompactness. Let $\mathbb{E}$ be a Banach space. By $\mathscr{M}$ we denote the set of all bounded subsets of $\mathbb{E}$. A function $\mu: \mathscr{M} \rightarrow \mathbb{R}_{+}$is called a measure of noncompactness if the following axioms are satisfied:

( $\mu 1) \quad \mu(X)=0 \Leftrightarrow \bar{X}$ is compact;

( $\mu 2) \quad \mu(\bar{X})=\mu(X)$;

( $\mu 3) \quad X \subseteq Y \Rightarrow \mu(X) \leq \mu(Y)$; 
( $\mu 4) \mu(\operatorname{conv}(X))=\mu(X)$;

( $\mu 5) \quad \mu(X \cup Y)=\max \{\mu(X), \mu(Y)\}$;

( $\mu 6) \quad \mu(r X)=|r| \mu(X) ; r \in \mathbb{R}$;

( $\mu 7) \quad \mu(X+Y) \leq \mu(X)+\mu(Y)$.

We remark that the above set of axioms is not minimal and any measure of noncompactness $\mu: \mathscr{M} \rightarrow \mathbb{R}_{+}$can be extended to the product $\mathbb{E} \times \mathbb{R}^{n}$ by $\mu(X)=\mu\left(\operatorname{pr}_{1}(X)\right)$, where $X \subset \mathbb{E} \times \mathbb{R}^{n}$ is a bounded subset and $\operatorname{pr}_{1}: \mathbb{E} \times \mathbb{R}^{n} \rightarrow \mathbb{E}$ denotes the projection on the first component. For more details concerning measures of noncompactness and examples we refer to [16, 24, 26, 28].

Let $X \subset \mathbb{E} \times \mathbb{R}^{n}$ be a subset and $F: X \rightarrow \mathbb{E}$ be a continuous map which sends bounded sets into bounded sets. $F$ is called a $\mu$-Lipschitzian map with a constant $k \geq 0$ if $\mu(F(A)) \leq k \mu(A)$, for all bounded $A \subset X$. A $\mu$ Lipschitzian map with constant $k=0$ is called a completely continuous map, and if $\mu(F(X))=0$, then $F$ is called compact. A $\mu$-Lipschitzian map with a constant $k<1$ is called a $\mu$-contraction map or a Darbo map. If $F$ is a $\mu$-Lipschitzian map with the constant 1 and $\mu(F(A))<\mu(A)$ for all bounded $A \subset X$ such that $\mu(A)>0$, then $F$ is called condensing.

Let $L: \operatorname{Dom}(L) \subseteq \mathbb{E} \rightarrow \mathbb{F}$ be a closed Fredholm operator of index zero. A compact resolvent of $L$ is a compact linear operator $K: \mathbb{E} \rightarrow \mathbb{F}$ such that $L+K: \operatorname{Dom}(L) \rightarrow \mathbb{F}$ is a bijection. By $\mathrm{CR}(L)$ we denote the set of all compact resolvents of $L$. For any $K \in \mathrm{CR}(L)$ we define $R_{K}:=(L+K)^{-1}: \mathbb{F} \rightarrow \mathbb{E}$. Since $L$ is a closed operator, $R_{K}$ is a bounded linear operator. One can easily observe that $\mathrm{CR}(L) \neq \varnothing$ and also, if $K_{1}, K_{2} \in \mathrm{CR}(L)$ and $R_{i}:=\left(L+K_{i}\right)^{-1}$; $i=1,2$, then

$$
\begin{gathered}
R_{1}\left(L+K_{2}\right)=\operatorname{Id}_{\mid \operatorname{Dom}(L)}+R_{1}\left(K_{2}-K_{1}\right) ; \\
R_{1}=R_{2}+R_{1}\left(K_{2}-K_{1}\right) R_{2},
\end{gathered}
$$

where $\operatorname{Id}_{\mid \operatorname{Dom}(L)}: \operatorname{Dom}(L) \rightarrow \operatorname{Dom}(L)$ denotes the identity map.

Let $X \subset \mathbb{E}$ be a bounded closed subset such that $\operatorname{Dom}(L) \cap X \neq \varnothing$ and assume that $B: X \rightarrow \mathbb{E}$ and $G: X \rightarrow \mathbb{F}$ are two mappings. We are going to study the following composite coincidence problem:

$$
\left\{\begin{array}{c}
\text { find } x \in X \text { such that } x-B(x) \in \operatorname{Dom}(L) \text { and } \\
L(x-B(x))=G(x) .
\end{array}\right.
$$

The problem $(\mathscr{P})$ is equivalent to the following fixed point problem:

$$
x=B(x)+R_{K}[G+K(I-B)](x)
$$

where $R_{K}=(L+K)^{-1}, K \in \mathrm{CR}(L)$. Indeed, the problem $(\mathscr{P})$ is equivalent to the equation

$$
L(x-B(x))+K(x-B(x))=G(x)+K(x-B(x))
$$

which can be transformed to (2.3).

Assume that $B: X \rightarrow \mathbb{E}$ and $G: X \rightarrow \mathbb{F}$ are two maps, and put

$$
\Theta_{K}(B, G):=B+R_{K}[G+K(I-B)]: X \rightarrow \mathbb{E} .
$$

The following problem is equivalent to $(\mathscr{P})$

$$
\left\{\begin{array}{c}
\text { find a fixed point } x \in X \text { of } \Theta_{K}(B, G) \text {, i.e. } \\
x=\Theta_{K}(B, G)(x), \quad x \in X .
\end{array}\right.
$$


Suppose that $K_{1}, K_{2} \in \mathrm{CR}(L), R_{i}:=\left(L+K_{i}\right)^{-1}, i=1,2$, then it follows from (2.2)

$$
\begin{aligned}
\Theta_{K_{1}}(B, G)= & \Theta_{K_{2}}(B, G)+R_{2}\left(K_{1}-K_{2}\right)(I-B) \\
& +R_{1}\left(K_{2}-K_{1}\right) R_{2}\left[G+K_{1}(I-B)\right]
\end{aligned}
$$

and consequently, by (2.2), we obtain

$$
\Theta_{K_{1}}(B, G)=\Theta_{K_{2}}(B, G)+R_{1}\left(K_{2}-K_{1}\right) \Theta_{K_{2}}(B, G)+R_{1}\left(K_{1}-K_{2}\right) .
$$

Now we can introduce the following definition

Definition 2.1. Let $B: X \rightarrow \mathbb{E}$ and $G: X \rightarrow \mathbb{F}$ be two maps. We say that the pair $(B, G)$ is an $L$-condensing pair if the map $\Theta_{K}(B, G): X \rightarrow \mathbb{E}, K \in \operatorname{CR}(L)$, is a condensing map.

It follows from (2.5) that the above definition does not depend on the choice of the resolvent $K \in \mathrm{CR}(L)$.

Definition 2.2. Let $(X, A)$ be a pair of closed bounded subsets of $\mathbb{E}$ such that $\operatorname{Dom}(L) \cap X \neq \varnothing$. We denote by $\mathscr{C}_{L}(X, A)$ the class of all $L$-condensing pairs $(B, G), B: X \rightarrow \mathbb{E}, G: X \rightarrow \mathbb{F}$ such that the problem $(\mathscr{P})$ has no solution in $A$, i.e. $x \neq \Theta_{K}(B, G)(x)$ for every $x \in A$.

The class $\mathscr{C}_{L}(X, A)$ can be equipped with a homotopy relation as follows: a path $\left(B_{t}, G_{t}\right) \in \mathscr{C}_{L}(X, A) ; t \in[0,1]$, is a homotopy in $\mathscr{C}_{L}(X, A)$ if the map $H: X \times[0,1] \rightarrow \mathbb{E}$ defined by $H(x, t)=\Theta_{K}\left(B_{t}, G_{t}\right)(x), x \in X, t \in$ $[0,1]$ is a condensing map. Moreover, if $(B, G)=\left(B_{0}, G_{0}\right)$ and $\left(B^{\prime}, G^{\prime}\right)=$ $\left(B_{1}, G_{1}\right)$, we say that $(B, G)$ and $\left(B^{\prime}, G^{\prime}\right)$ are homotopic in $\mathscr{C}_{L}(X, A)$ and we write $(B, G) \sim\left(B^{\prime}, G^{\prime}\right)$. It is easy to verify that this is an equivalence relation in $\mathscr{C}_{L}(X, A)$. The set of all homotopy classes in $\mathscr{C}_{L}(X, A)$ will be denoted by $\mathscr{C}_{L}[X, A]$. Let $\mathscr{C}(X, A)$ denote the class of all condensing maps $F: X \rightarrow \mathbb{E}$ such that $x \neq F(x)$ for every $x \in A$. The class $\mathscr{C}(X, A)$ can also be equipped with a homotopy relation, namely a path $F_{t} \in \mathscr{C}(X, A), t \in$ $[0,1]$, is a homotopy in $\mathscr{C}(X, A)$ if the map $H: X \times[0,1] \rightarrow E$ defined by $H(x, t)=F_{t}(x)$ is condensing. Similarly, the set of all homotopy classes in $\mathscr{C}(X, A)$ is denoted by $\mathscr{C}[X, A]$. From the definition, $\boldsymbol{\Theta}_{K}: \mathscr{C}_{L}(X, A) \rightarrow$ $\mathscr{C}(X, A)$ induces the map $\Theta_{K}^{*}: \mathscr{C}_{L}[X, A] \rightarrow \mathscr{C}[X, A]$. Since the map $\Theta_{K}$ transforms the composite coincidence problem $(\mathscr{P})$ into a fixed point problem $\left(\mathscr{P}^{\prime}\right)$ we call it a connection, (cf. [16]).

Definition 2.3. A pair $\left(B^{\prime}, G^{\prime}\right) \in \mathscr{C}_{L}(X, A)$ is called L-essential in $\mathscr{C}_{L}(X, A)$ if every pair $(B, G) \in \mathscr{C}_{L}(X, A)$, such that $B_{\mid A}^{\prime} \equiv B_{\mid A}$ and $G_{\mid A}^{\prime} \equiv G_{\mid A}$, has a solution in $X$ to the problem $(\mathscr{P})$, i.e. there exists a point $x \in X$ such that $x=\Theta_{K}(B, G)(x)$. Similarly, we say that $F \in \mathscr{C}(X, A)$ is essential in $\mathscr{C}(X, A)$ if every $F^{\prime} \in \mathscr{C}(X, A)$ such that $F_{\mid A} \equiv F_{\mid A}^{\prime}$ has a fixed point in $X$, i.e. there is $x \in X$ such that $x=F(x)$.

The topological transversality theorem for condensing maps (cf. [16]) says that if $F \sim F^{\prime}$ in $\mathscr{C}(X, A)$ and $F$ is an essential map then $F^{\prime}$ is also an essential map. This means that essentiality is a homotopy invariant. As we are going to show in the next theorem, this is also true for the class $\mathscr{C}_{L}(X, A)$. 
Theorem 2.4 (Topological Transversality Theorem). Suppose that $(B, G)$ and $\left(B^{\prime}, G^{\prime}\right)$ are two homotopic pairs in $\mathscr{C}_{L}(X, A)$. Then we have that $(B, G)$ is L-essential if and only if $\left(B^{\prime}, G^{\prime}\right)$ is L-essential.

Proof. Suppose that $\left(B^{\prime}, G^{\prime}\right)$ is not $L$-essential. Then, by definition, there is a pair $\left(B^{\prime \prime}, G^{\prime \prime}\right) \in \mathscr{C}_{L}(X, A)$ such that $B_{\mid A}^{\prime} \equiv B_{\mid A}^{\prime \prime}, G_{\mid A}^{\prime} \equiv G_{\mid A}^{\prime \prime}$ and $x \neq$ $\Theta_{K}\left(B^{\prime \prime}, G^{\prime \prime}\right)(x)$ for all $x \in X$. We put

$$
B_{t}^{\prime}:=t B^{\prime \prime}+(1-t) B^{\prime} \quad \text { and } \quad G_{t}^{\prime}:=t G^{\prime \prime}+(1-t) G^{\prime}, \quad t \in[0,1] .
$$

Since $\boldsymbol{\Theta}_{K}\left(B_{t}^{\prime}, G_{t}^{\prime}\right)=t \Theta_{K}\left(B^{\prime \prime}, G^{\prime \prime}\right)+(1-t) \Theta_{K}\left(B^{\prime}, G^{\prime}\right),\left(B_{t}^{\prime}, G_{t}^{\prime}\right) ; t \in[0,1]$ is a homotopy in $\mathscr{C}_{L}(X, A)$. Therefore we can assume that $(B, G)$ is homotopic to $\left(B^{\prime}, G^{\prime}\right)$ in $\mathscr{C}_{L}(X, A)$, where $x \neq \Theta_{K}\left(B^{\prime}, G^{\prime}\right)(x)$ for all $x \in X$. Let $\left(B_{t}, G_{t}\right)$ be a homotopy between $\left(B^{\prime}, G^{\prime}\right)$ and $(B, G)$. We define

$$
\begin{aligned}
N & :=\left\{x \in X: \text { there exists } t \in[0,1] \text { such that } L\left(I-B_{t}\right)(x)=G_{t}(x)\right\} \\
& =\left\{x \in X: \text { there exists } t \in[0,1] \text { such that } x=\Theta_{K}\left(B_{t}, G_{t}\right)(x)\right\} .
\end{aligned}
$$

It can be verified (see [16]) that $N$ is a closed subset of $X$ and $N \cap A=\varnothing$. We can assume without loss of generality that $N \neq \varnothing$ and let $\xi: X \rightarrow[0,1]$ be the Urysohn function such that $\xi_{\mid A} \equiv 1$ and $\xi_{\mid N} \equiv 0$. We put

$$
B^{*}(x):=B_{\xi(x)}(x) \text { and } G^{*}(x):=G_{\xi(x)}(x), \quad x \in X .
$$

Since a composition of a $\mu$-Lipschitzian map with constant 1 and a condensing map is a condensing map, it follows that $\left(B^{*}, G^{*}\right)$ is $L$-condensing. Moreover, if $x \in A$, then $\xi(x)=1$, thus $B^{*}(x):=B_{1}(x)=B(x)$ and $G^{*}(x)=$ $G_{1}(x)=G(x)$, and hence $B_{\mid A}^{*} \equiv B_{\mid A}$ and $G_{\mid A}^{*} \equiv G_{\mid A}$. We claim that $x \neq$ $\boldsymbol{\Theta}_{K}\left(B^{*}, G^{*}\right)(x)$, for every $x \in X$. Indeed, suppose that $x=\boldsymbol{\Theta}_{K}\left(B^{*}, G^{*}\right)(x)$, thus $x=\Theta_{K}\left(B_{\xi(x)}, G\right)_{\xi(x)}(x)$ and this implies that $x \in N$. But $\xi_{\mid N} \equiv 0$, therefore $x=\boldsymbol{\Theta}_{K}\left(B_{0}, G_{0}\right)(x)=\boldsymbol{\Theta}_{K}\left(B^{\prime}, G^{\prime}\right)(x)$ and this is a contradiction because we have assumed that $x \neq \boldsymbol{\Theta}_{K}\left(B^{\prime}, G^{\prime}\right)(x)$ for all $x \in X$. This implies that $(B, G)$ is not $L$-essential and the proof is completed.

Remark 2.5. Let us emphasize that the connection $\Theta_{K}$ satisfies the following properties:

(i) $(B, G) \sim\left(B^{\prime}, G^{\prime}\right)$ in $\mathscr{C}_{L}(X, A) \Rightarrow \Theta_{K}(B, G) \sim \Theta_{K}\left(B^{\prime}, G^{\prime}\right)$ in $\mathscr{C}(X, A)$;

(ii) $\boldsymbol{\Theta}_{K}(B, G)$ is essential in $\mathscr{C}(X, A) \Rightarrow(B, G)$ is $L$-essential in $\mathscr{C}_{L}(X, A)$.

Therefore in order to verify that a given pair $(B, G) \in \mathscr{C}_{L}(X, A)$ is $L$ essential it is sufficient to find an essential map in the homotopy class of the map $\boldsymbol{\Theta}_{K}(B, G)$. In the following, we will develop a topological degree theory to detect essential maps.

Let $\omega$ denote the class of all open bounded subsets in $\mathbb{E}$. Suppose that there is given a family of nonconstant functions $\left\{D_{\Omega}: \mathscr{E}[\bar{\Omega}, \partial \Omega] \rightarrow \mathbb{Z}\right\}_{\Omega \in \omega}$. For a condensing field $I-F: \bar{\Omega} \rightarrow \mathbb{E}$, where $F \in \mathscr{C}(\Omega, \partial \Omega)$ we define $d(I-F, \Omega):=$ $D_{\Omega}([F])$.

Definition 2.6. The function $d(\cdot, \Omega)$ is called a topological degree if the following condition is satisfied:

(D) (Additivity). For every $\Omega_{1}, \Omega_{2}, \Omega \in \omega$ such that $\Omega_{1} \cup \Omega_{2} \subset \Omega$ and $\Omega_{1} \cap \Omega_{2}=\varnothing$ and for every $F \in \mathscr{C}\left(\bar{\Omega}, \overline{\Omega \backslash\left(\Omega_{1} \cup \Omega_{2}\right)}\right)$, we have

$$
d(I-F, \Omega)=d\left(I-F, \Omega_{1}\right)+d\left(I-F, \Omega_{2}\right) .
$$


It is easy to verify that the property (D) implies the following properties:

Excision. If $F \in \mathscr{C}\left(\bar{\Omega}, \overline{\Omega \backslash \Omega_{1}}\right)$, where $\Omega_{1} \in \omega, \Omega_{1} \subset \Omega$, then $d\left(I-F, \Omega_{1}\right)$ $=d(I-F, \Omega)$.

Existence. Let $\Omega \neq \varnothing$. If $F \in \mathscr{C}(\bar{\Omega}, \partial \Omega)$ and $d(I-F, \Omega) \neq 0$, then $0 \in(I-F)(\Omega)$, i.e. there is $x_{0} \in \Omega$ such that $x_{0}=F\left(x_{0}\right)$.

Boundary Invariance. If $F, G \in \mathscr{C}(\bar{\Omega}, \partial \Omega)$ are such that $F_{\mid \partial \Omega} \equiv G_{\mid \partial \Omega}$, then $d(I-F, \Omega)=d(I-G, \Omega)$.

The last two properties imply that if $F \in \mathscr{C}(\bar{\Omega}, \partial \Omega)$ is such that $d(I-F, \Omega)$ $\neq 0$, then $F$ is essential in $\mathscr{C}(\bar{\Omega}, \partial \Omega)$. Suppose also the topological degree $d(\cdot, \Omega)$ satisfies the following property:

Normalization. Let $F: \bar{\Omega} \rightarrow\{*\} \subset \Omega$ be a constant map, then $d(I-F, \Omega)=$ 1 .

In this case, then the degree $d(\cdot, \Omega)$ is the well-known generalization of the Leray-Schauder degree to the class of condensing maps.

Now we introduce the definition of the composite coincidence degree. For every $L$-condensing pair $(B, G) \in \mathscr{C}_{L}(\bar{\Omega}, \partial \Omega), \Omega \in \omega$, we define

$$
d[(L, B, G), \Omega]:=d\left(I-\Theta_{K}(B, G), \Omega\right)
$$

where $K \in \mathrm{CR}(L)$. This definition depends on the choice of the resolvent $K$. In fact if $K_{1}, K_{2} \in \mathrm{CR}(L)$ and if $\operatorname{Id}+R_{1} \circ\left(K_{1}-K_{2}\right) \in G L_{c}^{+}(\mathbb{E})$, where $G L_{c}^{+}(\mathbb{E})$ denotes the connected component containing Id of the group $G L_{c}(\mathbb{E})$ of all isomorphisms of type $\mathrm{Id}+T$, where $T$ is a compact linear operator, then $d\left(I-\boldsymbol{\Theta}_{K_{1}}(B, G), \Omega\right)=d\left(I-\boldsymbol{\Theta}_{K_{2}}(B, G), \Omega\right)$, otherwise $d\left(I-\boldsymbol{\Theta}_{K_{1}}(B, G), \Omega\right)=$ $-d\left(I-\Theta_{K_{2}}(B, G), \Omega\right)$. This means that the above definition is unique up to sign for any choice of $K \in \mathrm{CR}(L)$.

\section{BASIC PROPERTIES, CONTINUATION THEOREM AND COMPUTATION OF THE COMPOSITE COINCIDENCE DEGREE}

Throughout this section we always assume that $L: \operatorname{Dom}(L) \subset \mathbb{E} \rightarrow \mathbb{F}$ is a Fredholm operator of index zero and $\Omega \subset \mathbb{E}$ is an open and bounded subset.

First of all, from the fundamental properties of the topological degree $d(\cdot, \Omega)$ and the properties of connection $\boldsymbol{\Theta}_{K}$ (cf. Remark (2.5)) we obtain the following:

Theorem 3.1. The composite coincidence degree satisfies the following properties:

(i) Additivity. If $\Omega_{1}$ and $\Omega_{2}$ are two disjoint subsets of $\Omega$ such that $(B, G) \in$ $\mathscr{C}_{L}\left(\bar{\Omega} ; \overline{\Omega \backslash\left(\Omega_{1} \cup \Omega_{2}\right)}\right)$, then

$$
d[(L, B, G), \Omega]=d\left[(L, B, G), \Omega_{1}\right]+d\left[(L, B, G), \Omega_{2}\right] .
$$

(ii) Excision. If $\Omega_{1} \subset \Omega$ is an open subset such that $(B, G) \in \mathscr{C}_{L}\left(\bar{\Omega}, \overline{\Omega \backslash \Omega_{1}}\right)$, then

$$
d[(L, B, G), \Omega]=d\left[(L, B, G), \Omega_{1}\right] .
$$

(iii) Existence. If $d[(L, B, G), \Omega] \neq 0$ then there exists $x \in \Omega$ such that $L(I-B)(x)=G(x)$.

(iv) Homotopy Invariance. If $\left(B_{t}, G_{t}\right) \in \mathscr{C}_{L}(\bar{\Omega}, \partial \Omega), t \in[0,1]$, is a homotopy in $\mathscr{C}_{L}(\bar{\Omega}, \partial \Omega)$, then $d\left[\left(L, B_{t}, G_{t}\right), \Omega\right]$ is independent of $t \in[0,1]$.

Proposition 3.2. Suppose that $P: \mathbb{E} \rightarrow \mathbb{E}$ and $Q: \mathbb{F} \rightarrow \mathbb{F}$ are bounded projections such that $\operatorname{Im} P=\operatorname{Ker} L$ and $\operatorname{Ker} Q=\operatorname{Im} L$. Let $T_{P}: \operatorname{Im} L \rightarrow \operatorname{Dom} L \cap \operatorname{Ker} P$ 
denotes the right inverse of $L$. Moreover, let $J: \operatorname{Im} Q \rightarrow \operatorname{Ker} L$ be an isomorphism. Then $K:=J^{-1} \circ P$ is a compact resolvent of $L$ and

$$
\Theta_{K}(B, G)=B+P(I-B)+J Q G+T_{P}(I-Q) G .
$$

Proof. Evidently $K:=J^{-1} \circ P$ is a linear operator with finite dimensional range, thus $K$ is compact. It is an easy observation that $L+K: \operatorname{Dom}(L) \rightarrow \mathbb{F}$ is a bijection and moreover $R_{K} Q y=J y$ and $R_{K}(I-Q) y=T_{P} y$, thus we have

$$
\begin{aligned}
\Theta_{K}(B, G) & =B+R_{K} \circ\left[G+J^{-1} P(I-B)\right] \\
& =B+R_{K}\left[Q G+(I-Q) G+J^{-1} P(I-B)\right] \\
& =B+J Q G+T_{P}(I-Q) G+P(I-B) .
\end{aligned}
$$

Proposition 3.3. Suppose that $B: X \rightarrow \mathbb{E}$ is a condensing map and $G: X \rightarrow \mathbb{F}$ is L-compact, i.e., $R_{K} \circ G: X \rightarrow \mathbb{F}$ is compact, then $(B, G)$ is an L-condensing pair.

Proof. We have $\Theta_{K}(B, G)=B+R_{K} \circ[G+K(I-B)]$, thus $\Theta_{K}(B, G)$ is a sum of the condensing map $B$ and the compact map $R_{K} \circ G+R_{K} \circ K \circ(I-B)$. Thus it is condensing.

Remark 3.4. According to the definition of $d[(L, B, G), \Omega]$ and Propositions 3.2 and 3.3 , we can easily verify the following relations:

(i) $d[(I, 0, G), \Omega]=d_{B}(I-G, \Omega)$ (Brouwer degree) if $\mathbb{E}=\mathbb{F}$ and $\operatorname{dim} \mathbb{E}$ $<\infty$

(ii) $d[(I, 0, G), \Omega]=d_{\text {L.S. }}(I-G, \Omega)$ (Leray-Schauder degree) if $\mathbb{E}=\mathbb{F}$ and $G$ is compact;

(iii) $d[(I, 0, G), \Omega]=d_{N}(I-G, \Omega)$ (Nussbaum degree) if $\mathbb{E}=\mathbb{F}$ and $G$ is a $\mu$-contractive map;

(iv) $d[(I, 0, G), \Omega]=d_{S}(I-G, \Omega)$ (Sadovskii degree) if $\mathbb{E}=\mathbb{F}$ and $G$ is a condensing map;

(v) $d[(L, 0, G), \Omega]=d_{M}[(L, G), \Omega]$ (Mawhin degree) if $G$ is an $L$ compact map;

(vi) $d[(L, B, G), \Omega]=d_{H}[(L, L B+G), \Omega]$ (Hetzer degree) if $B(\bar{\Omega}) \subseteq$ $\operatorname{Dom}(L)$ and $G$ is $L$-compact.

For details, we refer to $[2,12,13,17,20,23,24,25$ and 26].

As an immediate consequence of Theorem 3.1, Proposition 3.3 and Remark 3.5 , we get the following result which will be repeatedly used to reduce the solvability of boundary value problems of neutral equations to that of a corresponding retarded equation.

Corollary 3.5. If $G: \bar{\Omega} \times[0,1] \rightarrow \mathbb{F}$ is L-compact and $B: \bar{\Omega} \rightarrow \mathbb{F}$ is condensing such that for every $t \in[0,1],\left(t B, G_{t}\right) \in \mathscr{C}_{L}(\bar{\Omega}, \partial \Omega)$, where $G_{t}:=G(\cdot, t)$, then

$$
d\left[\left(L, B, G_{1}\right), \Omega\right]=d_{M}\left[\left(L, G_{0}\right), \Omega\right],
$$

where the latter is the Mawhin's coincidence degree of $\left(L, G_{0}\right)$ with respect to $\Omega$ (cf. [20]).

Proof. Since $\Theta\left(t B, G_{t}\right)=t \Theta\left(B, G_{t}\right)+(1-t) \Theta\left(0, G_{t}\right)$, by Proposition 3.3, $\left(t B, G_{t}\right), t \in[0,1]$ is a homotopy in $\mathscr{C}_{L}(\bar{\Omega}, \partial \Omega)$. Consequently, by Theorem (3.1) and (v) of Remark 3.4,

$$
d\left[\left(L, B, G_{1}\right), \Omega\right]=d\left[\left(L, 0, G_{0}\right), \Omega\right]=d_{M}\left[\left(L, G_{0}\right), \Omega\right] .
$$


It follows from the construction that $d_{M}\left[\left(L, G_{0}\right), \Omega\right]$, the Mawhin's coincidence degree, is equal to $d\left[\left(L, 0, G_{0}\right), \Omega\right]$.

The following result is a generalization of the antipodal theorem of Borsuk.

Corollary 3.6. Suppose that $\Omega$ is an open and bounded neighborhood of $0 \in \mathbb{E}$ which is symmetric with respect to 0 . Let $(B, G) \in \mathscr{C}_{L}(\bar{\Omega}, \partial \Omega)$ be such that $G(-x)=-G(x), B(-x)=-B(x)$ for $x \in \partial \Omega$, then $d[(L, B, G), \Omega]=1$ $(\bmod 2)$. In particular, if $G: \bar{\Omega} \times[0,1] \rightarrow \mathbb{F}$ is L-compact, $G_{0}(-x)=-G_{0}(x)$ for $x \in \partial \Omega$, and $B$ is condensing, such that $\left(t B, G_{t}\right) \in \mathscr{C}_{L}(\bar{\Omega}, \partial \Omega)$ for all $t \in[0,1]$, then

$$
d\left[\left(L, B, G_{1}\right), \Omega\right]=1 \quad(\bmod 2) .
$$

Proof. It follows immediately from the definition of $\Theta(B, G)$ that if both $B$ and $G$ are odd on $\partial \Omega$, then

$$
\boldsymbol{\Theta}(B, G)(-x)=-\Theta(B, G)(x), \quad x \in \partial \Omega .
$$

Therefore the result follows from the antipodal theorem of Sadovskii's degree. The second conclusion is a trivial consequence of Corollary (3.5).

The following result gives an extension of the Krasnosel'skii fixed point theorem.

Corollary 3.7. Suppose that $\Omega$ is an open and bounded neighborhood of $0 \in \mathbb{E}$ which is symmetric with respect to 0 . Assume that $(B, G) \in \mathscr{C}_{L}(\bar{\Omega}, \partial \Omega)$ and there is no point $x \in \partial \Omega$ such that $x-B x+\mu[x+B(-x)] \in \operatorname{Dom}(L)$ and

$$
L[x-B(x)+\mu(x+B(-x))]=G(x)-\mu G(-x)
$$

for some $\mu \in[0,1]$. Then the pair $(B, G)$ is L-essential in $\mathscr{C}_{L}(\bar{\Omega}, \partial \Omega)$, i.e. the composite coincidence problem

$$
L(I-B)(x)=G(x), \quad x \in \bar{\Omega},
$$

has a solution in $\Omega$.

Proof. Since $\boldsymbol{\Theta}(-B,-G)(-x)=-\Theta(B, G)(-x)$ for every $x \in \bar{\Omega}$, thus $\left(B^{\prime}, G^{\prime}\right)$, where $B^{\prime}(x):=-B(-x)$ and $G^{\prime}(x):=-G(-x)$, is also an $L$-condensing pair. For $\lambda \in[0,1]$ we define

$$
\begin{aligned}
& \widetilde{B}(x, \lambda):=\frac{1}{1+\lambda}[B(x)-\lambda B(-x)], \\
& \widetilde{G}(x, \lambda):=\frac{1}{1+\lambda}[G(x)-\lambda G(-x)]
\end{aligned}
$$

where $x \in \bar{\Omega}$. Since $\left(\widetilde{B}_{\lambda}, \widetilde{G}_{\lambda}\right)=\frac{1}{1+\lambda}(B, G)+\frac{\lambda}{1+\lambda}\left(B^{\prime}, G^{\prime}\right)$, where $\widetilde{B}_{\lambda}:=$ $\widetilde{B}(\cdot, \lambda), \widetilde{G}_{\lambda}:=\widetilde{G}(\cdot, \lambda)$, thus $\left(\widetilde{B}_{\lambda}, \widetilde{G}_{\lambda}\right)$ is an $L$-condensing pair for every $\lambda \in[0,1]$. We want to show that $\left(\widetilde{B}_{\lambda}, \widetilde{G}_{\lambda}\right)$ is a homotopy in $\mathscr{C}_{L}(\bar{\Omega}, \partial \Omega)$. Assume for contradiction that $\boldsymbol{\Theta}\left(\widetilde{B}_{\lambda}, \widetilde{G}_{\lambda}\right)(x)=x$ for some $(x, \lambda) \in \partial \Omega \times[0,1]$, then $L[x-\widetilde{B}(x, \lambda)]=\widetilde{G}(x, \lambda)$ i.e.

$$
L[(1+\lambda) x-B(x)+\lambda B(-x)]=G(x)-\lambda G(-x),
$$

then

$$
L[x-B(x)+\lambda(x+B(-x))]=G(x)-\lambda G(-x),
$$

which is a contradiction to the assumption. 
By the homotopy invariance property we get

$$
d\left[\left(L, \widetilde{B}_{1}, \widetilde{G}_{1}\right), \Omega\right]=d[(L, B, G), \Omega] .
$$

On the other hand $\widetilde{B}(x, 1)=\frac{1}{2}[B(x)-B(-x)]$ and $\widetilde{G}(x, 1)=\frac{1}{2}[G(x)-G(-x)]$ are odd mappings, thus by Corollary 3.5 ,

$$
d\left[\left(L, \widetilde{B}_{1}, \widetilde{G}_{1}\right), \Omega\right]=1(\bmod 2),
$$

consequently $d[(L, B, G), \Omega] \neq 0$ and therefore $(B, G)$ is an $L$-essential pair.

In what follows we assume that $P: \mathbb{E} \rightarrow \mathbb{E}$ and $Q: \mathbb{F} \rightarrow \mathbb{F}$ are two projections such that $\operatorname{Im} P=\operatorname{Ker} L$ and $\operatorname{Ker} Q=\operatorname{Im} L$. We also fix an isomorphism $J$ : $\operatorname{Im} Q \rightarrow \operatorname{Ker} L$. Then, by Proposition $3.2 K:=J^{-1} \circ P$ is a compact resolvent of $L$. We fix this resolvent and we will consider the composite coincidence degree with respect to the operator $K$. Moreover, by $T_{P}: \operatorname{Im} L \rightarrow \operatorname{Dom} L \cap \operatorname{Ker} P$ we denote the right inverse of $L$.

The following result gives an extension of the classical Leray-Schauder continuation theorem for a compact perturbation of the identity [17] and the generalized continuation theorem due to Mawhin (cf. [20, 21]) for coincidence degree.

Corollary 3.8. Suppose that $y \in \operatorname{Im} L$ is given. Let $B^{*}: \bar{\Omega} \times[0,1] \rightarrow \mathbb{E}$ be condensing and $G^{*}: \bar{\Omega} \times[0,1] \rightarrow \mathbb{F}$ be L-compact such that

(i) $B^{*}(\cdot, 1)=B, G^{*}(\cdot, 1)=G$;

(ii) For every $\lambda \in(0,1)$, the equation

$$
L\left[I-\lambda B^{*}(x, \lambda)\right]=\lambda G^{*}(x, \lambda)+y
$$

has no solution in $\partial \Omega$;

(iii) $Q G^{*}(x, 0) \neq 0$ for every $x \in L^{-1}\{y\} \cap \partial \Omega$;

(iv) $d_{B}\left(Q G^{*}(\cdot, 0)_{\mid L^{-1}\{y\}}, \Omega \cap L^{-1}\{y\}, 0\right) \neq 0$, where the last number denotes the Brouwer degree at $0 \in \operatorname{Im} Q$ of the continuous mapping $Q G^{*}(\cdot, 0)$ from the affine finite dimensional topological space $L^{-1}\{y\}$ into $\operatorname{Im} Q$.

Then the equation $L(I-B)(x)=G x+y$ has at least one solution in $\bar{\Omega}$.

Proof. Let $\widetilde{B}: \bar{\Omega} \times[0,1] \rightarrow \mathbb{E}$ and $\widetilde{G}: \bar{\Omega} \times[0,1] \rightarrow \mathbb{F}$ be defined as

$$
\widetilde{B}(x, \lambda)=\lambda B^{*}(x, \lambda) ; \quad \widetilde{G}(x, \lambda)=Q G^{*}(x, \lambda)+\lambda(I-Q) G^{*}(x, \lambda)+y
$$

for $x \in \bar{\Omega}$ and $\lambda \in[0,1]$. Evidently, $\widetilde{B}$ is condensing and $\widetilde{G}$ is $L$-compact. If for some $(x, \lambda) \in \bar{\Omega} \times(0,1), L(x-\widetilde{B}(x, \lambda))=\widetilde{G}(x, \lambda)$, that is

$$
L\left[x-\lambda B^{*}(x, \lambda)\right]=Q G^{*}(x, \lambda)+\lambda(I-Q) G^{*}(x, \lambda)+y .
$$

Therefore, by assumption (ii), $x \notin \partial \Omega$.

If $\lambda=0$ and $x \in \bar{\Omega}$ are given such that $L[x-\widetilde{B}(x, \lambda)]=\widetilde{G}(x, \lambda)$, i.e., $L x=$ $Q G^{*}(x, 0)+y$, thus $Q G^{*}(x, 0)=0$ and $L x=y$. Therefore, by assumption (iii), $x \notin \partial \Omega$. If $\lambda=1$ and $x \in \partial \Omega$ are given such that $L[x-\widetilde{B}(x, \lambda)]=$ $\widetilde{G}(x, \lambda)$, i.e. $L[x-B(x)]=Q G(x)+(I-Q) G(x)+y$, then $L(I-B)(x)=$ 
$G(x)+y$ has at least one solution in $\bar{\Omega}$. Therefore, if there exists no $x \in \partial \Omega$ such that $L(I-B)(x)=G(x)+y$, then the above argument indicates that

$$
L[I-\widetilde{B}(x, \lambda)] \neq \widetilde{G}(x, \lambda) \text { for } \lambda \in[0,1] \text { and } x \in \partial \Omega,
$$

thus $\left(\widetilde{B}_{\lambda}, \widetilde{G}_{\lambda}\right)$ is a homotopy in $\mathscr{C}_{L}(\bar{\Omega}, \partial \Omega)$ and, by the homotopy invariance and (v) of Remark 3.4, we obtain

$$
\begin{aligned}
d[(L, B, G), \Omega] & =d\left[\left(L, 0, \widetilde{G}_{0}\right), \Omega\right]=d\left[\left(L, 0, Q G^{*}(\cdot, 0)+y\right), \Omega\right] \\
& =d_{M}\left[\left(L, Q G^{*}(\cdot, 0)+y\right), \Omega\right] .
\end{aligned}
$$

On the other hand, by a result of Mawhin (cf. [20]), it follows that

$$
\left|d_{M}\left[\left(L, Q G^{*}(\cdot, 0)+y\right), \Omega\right]\right|=\left|d_{B}\left(Q G^{*}(\cdot, 0)_{\mid L^{-1}\{y\}}, \Omega \cap L^{-1}\{y\}, 0\right)\right| .
$$

Therefore assumption (iv) guarantees that $d[(L, B, G), \Omega] \neq 0$, hence the conclusion follows from the existence property of the composite coincidence degree.

Remark 3.9. The condition (iv) of Corollary (3.7) can be replaced by

$$
d_{B}\left(J Q G^{*}\left(\cdot+T_{P} y, 0\right)_{\mid \operatorname{Ker} L},\left(-T_{P} y+\Omega\right) \cap \operatorname{Ker} L, 0\right) \neq 0 .
$$

For illustrative purposes, in the remaining part of this section, we consider a periodic boundary value problem of neutral equations to show that the composite coincidence degree theory can be effectively applied to obtain some existence results for certain nonlinear problems.

Example 3.10. Let $C\left([a, b], \mathbb{R}^{n}\right)$ be the space of continuous functions from $[a, b]$ to $\mathbb{R}^{n}$ with the topology of uniform convergence. For a fixed $r \geq 0$, let $C=C\left([-r, 0], \mathbb{R}^{n}\right)$ with the norm $\|\varphi\|=\sup _{-r \leq \theta \leq 0}|\varphi(\theta)|$ for $\varphi \in C$. If $x \in C\left([\sigma-r, \sigma+\delta], \mathbb{R}^{n}\right)$ for some $\delta>0$ and $\sigma \in \mathbb{R}$, then $x_{t} \in C$ for $t \in[\sigma, \sigma+\delta]$ is defined by $x_{t}(\theta)=x(t+\theta)$ for $\theta \in[-r, 0]$.

We consider the following neutral functional differential equation

$$
\frac{d}{d t}\left[x(t)-b\left(t, x_{t}\right)\right]=f\left(t, x_{t}\right)
$$

where $f: \mathbb{R} \times C \rightarrow \mathbb{R}^{n}$ is completely continuous, $b: \mathbb{R} \times C \rightarrow \mathbb{R}^{n}$ is continuous, and there exists a constant $k<1$ such that

$$
|b(t, \varphi)-b(t, \psi)| \leq k\|\varphi-\psi\| \text { for } t \in \mathbb{R}, \varphi, \psi \in C .
$$

Assuming that $b(t+\omega, \varphi)=b(t, \varphi)$ and $f(t+\omega, \varphi)=f(t, \varphi)$ for $(t, \varphi) \in$ $\mathbb{R} \times C$ and some constant $\omega>0$, we want to find an $\omega$-periodic solution to the equation (3.1), namely, we want to find an $\omega$-periodic continuous function $x(t)$ such that $x(t)-b\left(t, x_{t}\right)$ is continuously differentiable and (3.1) holds for all $t \in \mathbb{R}$.

Proposition 3.11. Suppose that there exists a constant $M>0$ such that

(i) for any $\lambda \in(0,1)$ and any $\omega$-periodic solution $x$ of the system

$$
\frac{d}{d t}\left[x(t)-\lambda b\left(t, x_{t}\right)\right]=\lambda f\left(t, x_{t}\right),
$$

we have $|x(t)|<M$ for $t \in \mathbb{R}$; 
(ii) $g(u):=\frac{1}{\omega} \int_{0}^{\omega} f(s, \hat{u}) d s \neq 0$ for any $u \in \partial B_{M}\left(\mathbb{R}^{n}\right)$, where $B_{M}\left(\mathbb{R}^{n}\right)=$ $\left\{u \in \mathbb{R}^{n} ;|u|<M\right\}$ and $\hat{u}$ denotes the constant mapping from $[-r, 0]$ to $\mathbb{R}^{n}$ with the value $u \in \mathbb{R}^{n}$;

(iii) $d_{B}\left(g, B_{M}\left(\mathbb{R}^{n}\right), 0\right) \neq 0$.

Then there exists at least one $\omega$-periodic solution of the system (3.1) which satisfies $|x(t)|<M$ for $t \in \mathbb{R}$.

Proof. Let

$$
\begin{gathered}
P_{\omega}=\left\{h \in C\left(\mathbb{R}, \mathbb{R}^{n}\right) ; h(t+\omega)=h(t) \text { for } t \in \mathbb{R}\right\}, \\
H_{\omega}=\left\{H \in C\left(\mathbb{R}, \mathbb{R}^{n}\right) ; H(0)=0, H(t)=\alpha t+h(t)\right.
\end{gathered}
$$

for some $\alpha \in \mathbb{R}^{n}$ and $\left.h \in P_{\omega}\right\}$.

For any $h \in P_{\omega}$, let $\|h\|=\sup _{0<t<\omega}|h(t)|$ and, for any $H \in H_{\omega}, H(t)=$ $\alpha t+h(t), \alpha \in \mathbb{R}^{n}, h \in P_{\omega}$, let $\|\tilde{H}\|=|\alpha|+\|h\|$. It is easy to verify that $P_{\omega}$ and $H_{\omega}$ are Banach spaces.

We define the following mappings $L: P_{\omega} \rightarrow H_{\omega}, G: P_{\omega} \rightarrow H_{\omega}$, and $B: P_{\omega} \rightarrow$ $P_{\omega}$ as follows:

$$
\begin{gathered}
\operatorname{Lh}(t)=h(t)-h(0), \\
G h(t)=\int_{0}^{t} f\left(s, h_{s}\right) d s, \\
B h(t)=b\left(t, h_{t}\right), \quad h \in P_{\omega}, \quad t \in \mathbb{R},
\end{gathered}
$$

where $\operatorname{Im} G \subseteq H_{\omega}$, since $\int_{0}^{t} f\left(s, h_{s}\right) d s-\frac{t}{\omega} \int_{0}^{\omega} f\left(s, h_{s}\right) d s$ is an $\omega$-periodic function of $t \in \mathbb{R}$ for any $h \in P_{\omega}$.

It is easy to prove that $L$ is a continuous Fredholm operator of index zero, $B$ is a $k$-set contraction, $G$ is completely continuous and, finding an $\omega$-periodic solution of equation (3.1) is equivalent to solving the nonlinear problem $L(I-B)(x)=G(x)$.

On the other hand,

$\operatorname{Ker} L=\left\{h \in P_{\omega} ; h(t)=h(0)\right.$ for $\left.t \in \mathbb{R}\right\}, \quad \operatorname{Im} L=H_{\omega} \cap P_{\omega}$.

Define $P: P_{\omega} \rightarrow P_{\omega}$ and $Q: H_{\omega} \rightarrow H_{\omega}$ by

$$
\begin{gathered}
P h(t)=h(0), \quad h \in P_{\omega}, \quad t \in \mathbb{R}, \\
Q H(t)=\alpha t, \quad H \in H_{\omega}, \quad H(t)=\alpha t+h(t), \quad \alpha \in \mathbb{R}^{n}, \quad h \in P_{\omega}, \quad t \in \mathbb{R} .
\end{gathered}
$$

We can show that $P$ and $Q$ are projections and

$$
P_{\omega}=\operatorname{Ker} P \oplus \operatorname{Ker} L, \quad H_{\omega}=\operatorname{Im} L \oplus \operatorname{Im} Q .
$$

Let

$$
\Omega=\left\{x \in P_{\omega} ;|x(t)|<M \text { for } t \in \mathbb{R}\right\} .
$$

Evidently, the mapping $B^{*}: \bar{\Omega} \times[0,1] \rightarrow P_{\omega}$ defined by $B^{*}(h, \lambda)(t)=b\left(t, h_{t}\right)$ for $h \in P_{\omega}, t \in \mathbb{R}$ and $\lambda \in[0,1]$ is a $k$-set contraction and the mapping $G^{*}: \bar{\Omega} \times[0,1] \rightarrow H_{\omega}$ defined by $G^{*}(h, \lambda)(t)=\int_{0}^{t} f\left(s, h_{s}\right) d s$ for $h \in \bar{\Omega}$ and $t \in \mathbb{R}$ is $L$-compact. Since

By assumption (i), $L\left[I-\lambda B^{*}(x, \lambda)\right] \neq \lambda G^{*}(x, \lambda)$ for $(x, \lambda) \in \partial \Omega \times(0,1)$.

$$
Q G^{*}(h, 0)(t)=\frac{t}{\omega} \int_{0}^{\omega} f\left(s, h_{s}\right) d s, \quad h \in \bar{\Omega}, t \in \mathbb{R},
$$


assumption (ii) implies that

$$
Q G^{*}(h, 0) \neq 0 \text { for } h \in L^{-1}(0) \cap \partial \Omega .
$$

Obviously, the orientation preserving isomorphism $J$ between $\operatorname{Im} Q$ and $\operatorname{Ker} L$ can be chosen as the identity. Therefore from assumption (iii) it follows that

$$
d_{B}\left[\left.J Q G^{*}(\cdot, 0)\right|_{\operatorname{Ker} L}, \Omega \cap \operatorname{Ker} L, 0\right) \neq 0 .
$$

By Corollary 3.8 and Remark 3.9, the proof is completed.

Remark 3.12. We note that our result can be applied to the case where $b(t, \varphi)$ may not be differentiable in $t$ and may be nonlinear in $\varphi$, (for example,

$$
b(t, \varphi)=\frac{1}{2}\left|\sin \frac{2 \pi}{\omega} t\right| \cdot \frac{|\varphi(-r)|}{1+|\varphi(-r)|}
$$

for $(t, \varphi) \in \mathbb{R} \times C)$ which cannot be dealt with by methods employed in [5 and 11] (for the case where $b(t, \varphi)$ is linear in $\varphi$ ) and [13] (for the case where $b(t, \varphi)$ is differentiable in $t)$.

\section{A UNIFIED APPROACH EXTENDING EXISTENCE RESULTS OF BVPS FROM RETARDED EQUATIONS TO NEUTRAL EQUATIONS}

Encouraged by the example in the previous section, we will provide a unified approach extending results of boundary value problems for retarded equations to a class of neutrál equations.

We consider the following two-point boundary value problem

$$
\left\{\begin{array}{l}
\frac{d}{d t}\left[x(t)-b\left(t, x_{t}\right)\right]=f\left(t, x_{t}\right), \quad t \in[0, a], \\
M x_{0}+N x_{a}=\tilde{g}(x),
\end{array}\right.
$$

where

(H1) $a>0$ is a constant;

(H2) $b:[0, a] \times C \rightarrow \mathbb{R}^{n}$ is continuous and there exists a constant $k<1$ such that

$$
|b(t, \varphi)-b(t, \psi)| \leq k\|\varphi-\psi\| \text { for } t \in[0, a] \text { and } \varphi \in C
$$

(H3) $\tilde{g}: \mathbb{E}:=C\left([-r, a], \mathbb{R}^{n}\right) \rightarrow C$ is completely continuous;

(H4) $f:[0, a] \times C \rightarrow \mathbb{R}^{n}$ satisfies the following Carathéodory condition:

(H4a) for each $\varphi \in C$, the mapping $t \in[0, a] \rightarrow f(t, \varphi) \in \mathbb{R}^{n}$ is Lebesgue measurable;

(H4b) for almost each $t \in[0, a]$, the mapping $\varphi \in C \rightarrow f(t, \varphi) \in \mathbb{R}^{n}$, is continuous;

(H4c) for each $\rho>0$, there exists $\alpha_{\rho} \in L^{1}([0, a], \mathbb{R})$ such that, for almost every $t \in[0, a]$ and every $\varphi \in C$ with $\|\varphi\| \leq \rho$, one has

$$
|f(t, \varphi)| \leq \alpha_{\rho}(t) \text { for } t \in[0, a]
$$

(H5) $M: C \rightarrow C$, and $N: C \rightarrow C$ are bounded linear operators which satisfy:

(H5a) $\operatorname{Im}\left(M+N T_{a}\right)$ is bounded, where for each $t \in[0, a], T_{t}: C \rightarrow C$ is defined by

$$
\left(T_{t}, \varphi\right)(s)= \begin{cases}\varphi(t+s) & \text { if }-r \leq t+s \leq 0 \\ \varphi(0) & \text { if } 0<t+s \leq a, \varphi \in C\end{cases}
$$


(H5b) $\operatorname{dim} \operatorname{Ker}\left(M+N T_{a}\right)=\operatorname{codim} \operatorname{Im}\left(M+N T_{a}\right)<\infty$;

(H6) there exists a set-contractive mapping $p: \mathbb{E} \rightarrow C$ such that $p x(0)=$ $b\left(0, x_{0}\right)$ for $x \in \mathbb{E}$ and $M p+N \hat{b}: \mathbb{E} \rightarrow C$ is completely continuous, where $\hat{b}: \mathbb{E} \rightarrow C$ is defined by

$$
(\hat{b} x)(\theta)= \begin{cases}b\left(a+\theta, x_{a+\theta}\right), & -r \leq \theta \leq 0, a+\theta \geq 0, \\ p x(a+\theta), & -r \leq \theta \leq 0, a+\theta \leq 0, \quad x \in \mathbb{E} .\end{cases}
$$

Under the above assumptions, the boundary value problem (4.1) can be rewritten as

$$
\left\{\begin{array}{l}
\frac{d}{d t}\left[x(t)-b\left(t, x_{t}\right)\right]=f\left(t, x_{t}\right), \quad t \in[0, a], \\
M\left[\left(I-B_{b, p}\right) x\right]_{0}+N\left[\left(I-B_{b, p}\right) x\right]_{a}=g(x),
\end{array}\right.
$$

where

$$
g=\tilde{g}-(M p+N \hat{b})
$$

and $B_{b, p}: \mathbb{E} \rightarrow \mathbb{E}$ is defined by

$$
B_{b, p} x(t)= \begin{cases}p x(t), & t \in[-r, 0], \\ b\left(t, x_{t}\right), & t \in[0, a], x \in \mathbb{E} .\end{cases}
$$

Evidently, assumptions (H3) and (H6) imply that $g: \mathbb{E} \rightarrow C$ is completely continuous and assumptions (H2) and (H6) imply that $B_{b, p}: \mathbb{E} \rightarrow \mathbb{E}$ is a set-contraction.

We will show that reformulating the boundary value problem (4.1) in the form (4.2) depends on the choice of a suitable mapping $p: \mathbb{E} \rightarrow C$, and that because of few restrictions on $p$, the problem (4.2) is indeed sufficiently general.

We now consider an abstract formulation of the boundary value problem (4.2). Let $\mathbb{F}=L^{1}\left([0, a], \mathbb{R}^{n}\right) \times C$ and $R: \mathbb{E} \rightarrow C\left([0, a] ; \mathbb{R}^{n}\right)$ be the restriction operator from $[-r, a]$ to $[0, a]$. Define

Dom $L=\{x \in \mathbb{E} ; R x$ is absolutely continuous on $[0, a]\}$,

$$
\begin{aligned}
L x & =\left((R x)^{\prime}, M x_{0}+N x_{a}\right), \quad x \in \operatorname{Dom} L, \\
F x(t) & =f\left(t, x_{t}\right), \quad t \in[0, a], \quad x \in \mathbb{E}, \\
G x & =(F x, g x), \quad x \in \mathbb{E} .
\end{aligned}
$$

Then $L: \operatorname{Dom} L \subseteq \mathbb{E} \rightarrow \mathbb{F}, F: \mathbb{E} \rightarrow L^{1}\left([0, a], \mathbb{R}^{n}\right), G: \mathbb{E} \rightarrow \mathbb{F}$, and $x$ is a solution of the boundary value problem (4.2) iff $x$ solves the following abstract operator equation

$$
L\left(I-B_{b, p}\right) x=G x .
$$

It has been shown in $\S 1$ of Mawhin [20] that $L$ is a Fredholm operator of index zero and $G$ is $L$-compact. Moreover, we have shown that $B_{b, p}$ is a $k$-set contraction. As an immediate consequence of Theorem 3.1, we obtain the following general existence theorem.

Theorem 4.1. Suppose that assumptions $(\mathrm{H1})-(\mathrm{H} 6)$ are satisfied. If there exists an open bounded set $\Omega \subseteq \mathbb{E}$ such that the boundary value problem (4.2) has no solution in $\partial \Omega$ and $d\left[\left(L, B_{b, p}, G\right), \Omega\right] \neq 0$, then (4.2) has at least one solution in $\Omega$.

Application of this general result requires solving two difficult problemsfinding a priori bounds and estimating $d\left[\left(L, B_{b, p}, G\right), \Omega\right]$. The major purpose 
of this section is to apply Corollary 3.5 to the boundary value problem (4.2) so that this problem can be reduced to a corresponding problem for an "associated" retarded equation.

We consider the following family of boundary value problem

$$
\left\{\begin{array}{l}
\frac{d}{d t}\left[x(t)-\lambda b\left(t, x_{t}\right)\right]=f^{*}\left(t, x_{t}, \lambda\right), \\
M\left[\left(I-\lambda \beta_{b, p}\right) x\right]_{0}+N\left[\left(I-\lambda B_{b, p}\right) x\right]_{a}=g^{*}(x, \lambda),
\end{array}\right.
$$

where

(H7) $g^{*}: \mathbb{E} \times[0,1] \rightarrow C$ is completely continuous and $g^{*}(x, 1)=g(x)$ for $x \in \mathbb{E}$,

(H8) $f^{*}:[0, a] \times C \times[0,1] \rightarrow \mathbb{R}^{n}$ satisfies the following Carathéodory condition

(H8a) for each $(\varphi, \lambda) \in C \times[0,1]$, the mapping $t \in[0, a] \rightarrow f^{*}(t, \varphi, \lambda) \in \mathbb{R}^{n}$ is Lebesgue measurable;

(H8b) for almost each $t \in[0, a]$, the mapping

$$
(\varphi, \lambda) \in C \times[0,1] \rightarrow f^{*}(t, \varphi, \lambda) \in \mathbb{R}^{n}
$$

is continuous;

(H8c) for each $\rho>0$, there exists $\alpha_{\rho} \in L^{1}([0, a], \mathbb{R})$ such that $\left|f^{*}(t, \varphi, \lambda)\right|$ $\leq \alpha_{\rho}(t)$ a.e. on $[0, a]$ for every $(\varphi, \lambda) \in C \times$
$(\mathrm{H} 8 \mathrm{~d}) f^{*}(t, \varphi, 1)=f(t, \varphi)$ for $(t, \varphi) \in[0, a] \times C$.

Define

$$
\widetilde{G}: \mathbb{E} \times[0,1] \rightarrow L^{1}\left([0, a], \mathbb{R}^{n}\right) \times C
$$

and

$$
F^{*}: \mathbb{E} \times[0,1] \rightarrow L^{1}\left([0, a], \mathbb{R}^{n}\right)
$$

as follows

$$
\begin{gathered}
F^{*}(x, \lambda)(t)=f^{*}\left(t, x_{i}, \lambda\right), \\
\widetilde{G}^{*}(x, \lambda)=\left(F^{*}(x, \lambda), g^{*}(x, \lambda)\right), \quad x \in \mathbb{E}, \lambda \in[0,1], t \in[0, a] .
\end{gathered}
$$

Then the boundary value problem (4.4) is equivalent to the following family of nonlinear problems:

$$
L\left(I-\lambda B_{b, p}\right) x=\widetilde{G}(x, \lambda), \quad \lambda \in[0,1] .
$$

Evidently, if $\lambda=0$, we obtain the following boundary value problem of retarded equation

$$
\left\{\begin{array}{l}
\dot{x}(t)=f^{*}\left(t, x_{t}, 0\right), \\
M x_{0}+N x_{a}=g^{*}(x, 0),
\end{array}\right.
$$

which has been extensively studied in the literature (see, e.g. [7 and 20]). Therefore the following reduction is significant.

Corollary 4.1. If there exists an open bounded set $\Omega \subseteq \mathbb{E}$ such that for each $\lambda \in[0,1]$, problem (4.4) has no solution on $\partial \Omega$, and Mawhin's coincidence degree $d_{M}[(L, \widetilde{G}(\cdot, 0)), \Omega] \neq 0$, then the problem (4.2) has at least one solution in $\Omega$.

The proof is a direct application of Corollary 3.5 and the existence property of the generalized coincidence degree. 
Remark 4.1. In [5], we developed a homotopy invariance property of Mawhin's coincidence degree which allows a continuous deformation of the linear part. This homotopy invariance was applied to reduce a periodic boundary value problem of neutral equation (4.1) with $b(t, \varphi)$ linear in $\varphi$ to a corresponding problem of a retarded equation. Corollary 4.1 extends this result to a more general case where $b(t, \varphi)$ may be nonlinear in $\varphi$ and the boundary value condition is more general.

To obtain an application of the continuation theorem (Corollary 3.8) to the boundary value problem (4.1), we denote by $P_{1}$ and $P_{2}$ the projection in $C$ such that $\operatorname{Im} P_{1}=\operatorname{Ker}\left(M+N T_{a}\right)$ and $\operatorname{Im} P_{2}=\operatorname{Im}\left(M+N T_{a}\right)$. Then by $\S 1$ in [20], the projector $Q: \mathbb{F} \rightarrow \mathbb{F}$ such that $\operatorname{Ker} Q=\operatorname{Im} L$ can be chosen as

$$
Q(h, c)=\left(0,\left(I-P_{2}\right)\left(c-N S_{a} h\right)\right), \quad(h, c) \in \mathbb{F},
$$

where for each $t \in[0, a], S_{t}: L^{1}\left([0, a], \mathbb{R}^{n}\right) \rightarrow C$ is defined as

$$
\left(S_{t} h\right)(s)= \begin{cases}\int_{0}^{t+s} h(u) d u, & 0 \leq t+s \leq a, \\ 0, & -r \leq t+s \leq 0, s \in[-r, 0] .\end{cases}
$$

Moreover, it is easy to verify that

$\operatorname{Ker} L=\left\{x \in \mathbb{E} ; x_{0} \in \operatorname{Ker}\left(M+N T_{a}\right), x(t)=x(0)\right.$ for $\left.t \in[0, a]\right\}$.

By Corollary 3.8, we obtain the following result:

Corollary 4.4. Suppose that there exist mappings $b^{*}:[0, a] \times C \times[0,1] \rightarrow \mathbb{R}^{n}$, $f^{*}:[0, a] \times C \times[0,1] \rightarrow \mathbb{R}^{n}$ and $g^{*}: \mathbb{E} \times[0,1] \rightarrow C$ such that

(i) $b^{*}(t, \varphi, 1)=b(t, \varphi), f^{*}(t, \varphi, 1)=f(t, \varphi), g^{*}(x, 1)=g(x)$ for $(t, x, \varphi) \in[0, a] \times \mathbb{E} \times C$;

(ii) $b^{*}$ is continuous and there exists a constant $k<1$ such that

$\left|b^{*}(t, \varphi, \lambda)-b^{*}(t, \psi, \lambda)\right| \leq k\|\varphi-\psi\|, \quad(t, \varphi, \psi, \lambda) \in[0, a] \times C \times C \times[0,1] ;$

(iii) $g^{*}$ and $f^{*}$ satisfy $(\mathrm{H} 7)$ and $(\mathrm{H} 8)$;

(iv) for each $\lambda \in(0,1)$, the boundary value problem

$$
\left\{\begin{array}{l}
\frac{d}{d t}\left[x(t)-\lambda b^{*}\left(t, x_{t}, \lambda\right)\right]=\lambda f^{*}\left(t, x_{t}, \lambda\right), \\
M\left[\left(I-\lambda B_{b_{\lambda}^{*}, p_{\lambda}^{*}}\right) x\right]_{0}+N\left[\left(I-\lambda B_{b_{\lambda}^{*}, p_{\lambda}^{*}}\right) x\right]_{1}=\lambda g^{*}(x, \lambda),
\end{array}\right.
$$

has no solution in $\partial \Omega$, where $b_{\lambda}^{*}(t, \varphi)=b^{*}(t, \varphi, \lambda)$ for $(t, \varphi, \lambda) \in[0, a] \times$ $C \times[0,1]$ and $p_{\lambda}^{*}: \mathbb{E} \rightarrow C$ is given such that $p^{*}(x)(0)=b^{*}\left(0, x_{0}, \lambda\right)$ and the mapping $(\lambda, x) \in[0,1] \times \mathbb{E} \rightarrow \rho_{\lambda}^{*}(x) \in C$ is a $k$-set contraction;

(v) $\left(I-P_{2}\right)\left(g-N S_{a} F\right) x \neq 0$ for $x \in \partial \Omega \cap \operatorname{Ker} L$;

(vi) $d_{B}\left(\left.J\left(I-P_{2}\right)\left(g-N S_{a} F\right)\right|_{\operatorname{Ker} L}, \Omega \cap \operatorname{Ker} L, 0\right) \neq 0$.

Then the boundary value problem (4.2) has at least one solution in $\Omega$.

Example 4.5. For the purpose of illustration, we apply the above results to the periodic boundary value problem

$$
\left\{\begin{array}{l}
\frac{d}{d t}\left[x(t)-b\left(t, x_{t}\right)\right]=f\left(t, x_{t}\right), \quad 0 \leq t \leq a \\
x_{0}=x_{a}
\end{array}\right.
$$

where $a \geq r$ is a given constant, $b$ and $f$ satisfy the assumptions (H2) and (H4). It is easy to verify assumptions (H3) and (H5) with $\tilde{g} \equiv 0, M=\mathrm{Id}$ and $N=-$ Id . 
Define $p: \mathbb{E} \rightarrow C$ by

$$
p x(\theta)=b\left(0, x_{0}\right)-b\left(a, x_{a}\right)+\hat{b}(x)(\theta) \text { for } \theta \in[-r, 0] \text { and } x \in \mathbb{E} \text {. }
$$

Then $p x(0)=\hat{b}(x)(0)+b\left(0, x_{0}\right)-b\left(a, x_{a}\right)=b\left(0, x_{a}\right)$ and $(M p+N \hat{b}) x=$ $p x-\hat{b} x$ that is

$$
\begin{aligned}
((M p+N \hat{b}) x)(\theta) & =b\left(0, x_{0}\right)-b\left(a, x_{a}\right)+\hat{b}(x)(\theta)-\hat{b}(x)(\theta) \\
& =b\left(0, x_{0}\right)-b\left(a, x_{a}\right) .
\end{aligned}
$$

Because of the set-contraction of $b, M p+N \hat{b}: \mathbb{E} \rightarrow C$ is completely continuous. This verifies (H6).

Because of $M=$ Id and $N=-$ Id, we have

$$
\operatorname{Ker}\left(M+N T_{1}\right)=\{\varphi \in C ; \varphi(\theta)=\varphi(0) \text { for } \theta \in[-r, 0]\}
$$

and $\operatorname{Im}\left(M+N T_{a}\right)=\{\varphi \in C ; \varphi(0)=0\}$. We define the projectors $P_{1}$ and $P_{2}$ as follows

$$
P_{1} \varphi(\theta)=\varphi(0), \quad P_{2} \varphi(\theta)=\varphi(\theta)-\varphi(0)
$$

for $\varphi \in C$ and $\theta \in[-r, 0]$. Then $\left(I-P_{2}\right)\left(g+S_{a} F\right) x \neq 0$ is equivalent to $g x(0)+S_{a} F x(0) \neq 0$, that is,

$$
b\left(a, x_{a}\right)-b\left(0, x_{0}\right)+\int_{0}^{a} f\left(u, x_{u}\right) d u \neq 0,
$$

where $g: \mathbb{E} \rightarrow C$ is defined by

$$
g x(\theta)=b\left(a, x_{a}\right)-b\left(0, x_{0}\right), \quad x \in \mathbb{E}, \theta \in[-r, 0] .
$$

On the other hand,

$$
\operatorname{Ker} L=\{x \in \mathbb{E} ; x(\theta)=x(0) \text { for } \theta \in[-r, a]\} \text {. }
$$

Therefore

$$
\left(I-P_{2}\right)\left(g+S_{a} F\right) x \neq 0 \text { for } x \in \operatorname{Ker} L
$$

is equivalent to

$$
b(a, \widehat{x(0)})-b(0, \widehat{x(0)})+\int_{0}^{a} f(u, \widehat{x(0)}) d u \neq 0 \quad \text { for } x(0) \in \mathbb{R}^{n}
$$

By Corollary 4.4, we obtain the following

Proposition 4.6. Suppose that there exists a constant $M>0$ such that

(i) for each $\lambda \in(0,1)$, any solution of the boundary value problem

$$
\left\{\begin{array}{l}
\frac{d}{d t}\left[x(t)-\lambda b\left(t, x_{t}\right)\right]=\lambda f\left(t, x_{t}\right), \\
x_{0}=x_{1}
\end{array}\right.
$$

satisfies $|x(t)|<M$ for $t \in[-r, a]$

(ii) $W(u):=b(a, \hat{u})-b(0, \hat{u})+\int_{0}^{a} f(\theta, \hat{u}) d \theta \neq 0$ for all $u \in \partial B_{M}\left(\mathbb{R}^{n}\right)$;

(iii) $d_{B}\left(W, B_{M}\left(\mathbb{R}^{n}\right), 0\right) \neq 0$.

Then the periodic boundary value problem (4.9) has at least one solution which satisfies $|x(t)| \leq M$ for $t \in[-r, a]$.

Clearly, applications of Corollary 4.2 and Corollary 4.4 require a priori bounds for the solutions of the problem (4.1). In the following part, we show that the existence of guiding functions implies certain a priori bounds for solutions. 
Definition 4.7. A continuously differentiable function $V: \mathbb{R}^{n} \rightarrow \mathbb{R}^{1}$ is said to be a guiding function for the boundary value problem (4.1) if there exists $\rho>0$ such that for every $\lambda \in(0,1)$ and every solution of the boundary value problem

$$
\left\{\begin{array}{l}
\frac{d}{d t}\left[x(t)-\lambda b\left(t, x_{1}\right)\right]=\lambda f\left(t, x_{1}\right), \quad t \in[0, a], \\
M\left[\left(I-\lambda B_{b, p}\right) x\right]_{0}+N\left[\left(I-\lambda B_{b, p}\right) x\right]_{a}=\lambda g(x),
\end{array}\right.
$$

we have

(i) for every $t \in(0, a)$ with $\left|x(t)-\lambda b\left(t, x_{t}\right)\right| \geq \rho$ and $\left|V\left(x(t)-\lambda b\left(t, x_{t}\right)\right)\right|$ $\geq\left|V\left(x(s)-\lambda b\left(x, s_{s}\right)\right)\right|$ for $s \in[0, a]$, it follows that

$$
\left\langle\operatorname{grad} V\left(x(t)-\lambda b\left(t, x_{t}\right)\right), f\left(t, x_{t}\right)\right\rangle>0 ;
$$

(ii) if $\left|x(a)-\lambda b\left(a, x_{a}\right)\right| \geq \rho$ and $\left|V\left(x(a)-\lambda b\left(a, x_{a}\right)\right)\right| \geq$ $\left|V\left(x(s)-\lambda b\left(s, x_{s}\right)\right)\right|$ for $s \in[0, a]$, then

$$
V\left(x(a)-\lambda b\left(a, x_{a}\right)\right)\left\langle\operatorname{grad} V\left(x(t)-\lambda b\left(a, x_{a}\right)\right), f\left(a, x_{a}\right)\right\rangle<0 ;
$$

(iii) if $\left|x(0)-\lambda b\left(0, x_{0}\right)\right| \geq \rho$ and $\left|V\left(x(0)-\lambda b\left(0, x_{0}\right)\right)\right| \geq\left|V\left(x(s)-\lambda b\left(s, x_{s}\right)\right)\right|$ for $s \in[0, a]$, then

$$
V\left(x(0)-\lambda b\left(0, x_{0}\right)\right)\left\langle\operatorname{grad} V\left(x(0)-\lambda b\left(0, x_{0}\right)\right), f\left(0, x_{0}\right)\right\rangle>0 \text {. }
$$

Theorem 4.8. Suppose that

(i) there exist $m+1$ guiding functions $V_{0}, V_{1}, \ldots, V_{m}$ such that for any $\lambda \in(0,1)$ and solution $x(t)$ of $(4.10)$,

$$
\lim _{|x| \rightarrow \infty} \sup \left\{\sum_{j=0}^{m}\left|V_{j}\left(x(t)-\lambda b\left(t, x_{t}\right)\right)\right| ; t \in[0, a]\right\}=\infty,
$$

where $|x|=\sup _{-r \leq \theta \leq a}|x(\theta)|$,

(ii) there exists a sufficiently large $\rho^{*}>0$ such that if $\tilde{\rho}>\rho^{*}$, then $\left(I-P_{2}\right)\left(g-N S_{a} F\right) x \neq 0$ for $x \in \partial \Omega_{\tilde{\rho}} \cap \operatorname{Ker} L$ and

$$
d_{B}\left(\left.J\left(I-P_{2}\right)\left(g-N S_{a} F\right)\right|_{\operatorname{Ker} L}, \Omega_{\tilde{\rho}} \cap \operatorname{Ker} L, 0\right) \neq 0,
$$

where $\Omega_{\tilde{\rho}}=\{x \in \mathbb{E}:|x(t)|<\tilde{\rho}$ for $t \in[-r, a]\}$. Then the boundary value problem (4.1) has at least one solution.

Proof. We write $\rho_{j}$ for the corresponding value of $\rho$ of the guiding function $V_{j}$. For fixed $\lambda \in(0,1), j=0,1, \ldots, m$, and a given solution of (4.10), we consider the function $W_{j}(t)=\left[V_{j}\left(x(t)-\lambda b\left(t, x_{t}\right)\right)\right]^{2}$ on $[0, a]$. If $W_{j}(t)$ attains its maximum at a $t \in(0, a)$, then

$$
W_{j}^{\prime}(t)=2 V_{j}\left(x(t)-\lambda b\left(t, x_{t}\right)\right)\left\langle\operatorname{grad} V_{j}\left(x(t)-\lambda b\left(t, x_{t}\right)\right), f\left(t, x_{t}\right)\right\rangle=0 .
$$

Therefore from (i) of the definition of a guiding function it follows that $\mid x(t)-$ $\lambda b\left(t, x_{t}\right) \mid<p_{j}$. If $W_{j}(t)$ attains its maximum at $t=a$, then $W_{j}^{\prime}(a) \geq 0$ which, by the assumption (ii) of a guiding function, implies that $\left|x(a)-\lambda b\left(a, x_{a}\right)\right|<$ $\rho_{j}$. Likewise, we can prove that if $W_{j}(t)$ attains its maximum at $t=0$, then $\left|x(0)-\lambda b\left(0, x_{0}\right)\right|<\rho_{j}$. Therefore, we obtain

$$
W_{j}(t) \leq \sup _{|x| \leq \rho_{j}}\left|V_{j}(x)\right|^{2} \triangleq M_{j}^{2} \quad \text { for } t \in[0, a]
$$


from which it follows that $\left|V_{j}\left(x(t)-\lambda b\left(t, x_{t}\right)\right)\right| \leq M_{j}$ and

$$
\sum_{j=0}^{m}\left|V_{j}\left(x(t)-\lambda b\left(t, x_{t}\right)\right)\right| \leq \sum_{j=0}^{m} M_{j} \text { for } t \in[0, a] .
$$

This implies, by assumption (4.11), that there exists a constant $\rho=\rho\left(\sum_{j=0}^{m} M_{j}\right)$ such that $|x(t)| \leq \rho\left(\sum_{j=0}^{m} M_{j}\right)$ for $t \in[-r, a]$. Therefore, our conclusion follows from Corollary 4.4 with

$$
\Omega=\left\{x \in \mathbb{E}:|x(t)|<\max \left\{\rho^{*}, \rho\left(\sum_{j=0}^{m} M_{j}\right)\right\}+1 \text { for } t \in[-r, a]\right\} .
$$

Applying the above result to the periodic boundary value problem (4.9), we obtain the following

Proposition 4.9. Suppose that

(i) $r=a, b(t+a, \varphi)=b(t, \varphi)$ for $(t, \varphi) \in \mathbb{R} \times C$ and $b(t, \varphi)$ is Lipschitz in $\varphi$ with a Lipschitz constant $k<1$;

(ii) there exist $m+1$ guiding functions $V_{0}, V_{1}, \ldots, V_{m}$ for the problem (4.9) such that $\lim _{|x| \rightarrow \infty}\left[\left|V_{0}(x)\right|+\cdots+\left|V_{m}(x)\right|\right]=\infty$;

(iii) $\left\langle\operatorname{grad} V_{0}(u), f(t, \hat{u})\right\rangle>0$ for all $u \in R^{n}$ with $|u| \geq \bar{\rho}$, where $\bar{\rho}$ is a constant.

Then the periodic boundary value problem (4.9) has at least one solution.

Proof. For any $\lambda \in(0,1)$ and any solution $x(t)$ of the boundary value problem

$$
\left\{\begin{array}{l}
\frac{d}{d t}\left[x(t)-\lambda b\left(t, x_{t}\right)\right]=\lambda f\left(t, x_{t}\right), \\
x_{0}=x_{a}
\end{array}\right.
$$

let $y(t)=x(t)-\lambda b\left(t, x_{t}\right)$. Suppose that there exists a constant $N>0$ such that $|y(t)| \leq N$ for $t \in[0, a]$. Then by the Lipschitz condition of $b(t, \varphi)$ in $\varphi$, we get

$$
|x(t)| \leq N+\sup _{t \in[0, a]}|b(t, 0)|+k\left\|x_{t}\right\| .
$$

If there exists $t^{*} \in[0, a]$ such that $\left|x\left(t^{*}\right)\right|=\max _{t \in[0, a]}|x(t)|$, then because of $x_{0}=x_{a}$ and $r=a$, we have $\left|x\left(t^{*}\right)\right|=\sup _{-r \leq t \leq a}|x(t)|$ and thus from (4.13) it follows that

$$
|x(t)| \leq\left|x\left(t^{*}\right)\right| \leq \frac{N+\sup _{t \in[0, a]}|b(t, 0)|}{1-k}
$$

for $t \in[0, a]$. This proves that if $\sup _{-r \leq \theta \leq a}|x(\theta)| \rightarrow \infty$, then

$$
\sup _{t \in[0, a]}\left|x(t)-\lambda b\left(t, x_{t}\right)\right| \rightarrow \infty .
$$

Consequently, condition (ii) implies (4.11).

On the other hand, by assumption (iii), $d_{B}\left(W, B_{\bar{\rho}}\left(\mathbb{R}^{n}\right), 0\right) \neq 0$, where $W: \mathbb{R}^{n} \rightarrow \mathbb{R}^{n}$ is defined as $W(u)=\int_{0}^{a} f(\theta, \hat{u}) d \theta$. Our conclusion then follows from Proposition 4.6 and Theorem 4.8. 


\section{REFERENCES}

1. S. R. Bernfeld, G. Ladde and V. Lakshmikantham, Nonlinear boundary value problems and several Lyapunov functions for functional differential equations, Boll. Un. Mat. Ital. 10 (1974), 602-613.

2. L. E. J. Brouwer, Über Abbildung von Mannigfaltigkeiten, Math. Ann. 71 (1972), 97-115.

3. J. Cronin, Periodic solutions of nonautonomous equations, Boll. Un. Math. Ital. (4) 6 (1972), 45-54.

4. $\ldots$, Equations with unbounded nonlinearities, J. Differential Equations 14 (1973), 581596.

5. L. H. Erbe, W. Krawcewicz, and J. H. Wu, Leray-Shauder degree for semilinear Fredholm maps and periodic boundary value problems of neutral equations, Nonlinear Analysis, TMA 15 (1990), 747-764.

6. M. Faheem and R. M. Rao, A boundary value problem for functional differential equations of neutral type, J. Math. Phys. Sci (4) 18 (1984), 381-404.

7. R. E. Gaines and J. Mawhin, Coincidence degree and nonlinear differential equations, Lecture Notes in Math., vol. 568, Springer-Verlag, Berlin and New York, 1977.

8. A. Granas, The theory of compact vector fields and some of its applications to the topology of functional spaces. (I), Rozprawy Mat. 30 (1962), 1-93.

9. G. B. Gustafson and K. Schmitt, Periodic solutions of hereditary differential equations, J. Differential Equations 13 (1977), 567-587.

10. _ A note on periodic solutions for delay-differential systems, Proc. Amer. Math. Soc. 42 (1974), 161-166.

11. J. K. Hale and J. Mawhin, Coincidence degree and periodic solutions of neutral equations, J. Differential Equations 15 (1974), 295-307.

12. G. Hetzer, Some remarks on $\phi_{t}$ on operators and on the coincidence degree for a Fredholm equation with noncompact nonlinear perturbations, Ann. Soc. Sci. Bruxelles Ser. I 89 (1975), 497-508.

13. __ Some applications of the coincidence degree for set-contractions to functional differential equations of neutral type, Comment. Math. Univ. Carolin. 16 (1975), 121-138.

14. M. A. Krasnosel'skii, The operator of translation along trajectories of ordinary differential equations, Amer. Math. Soc., Providence, R.I., 1968.

15. M. A. Krasnosel'skii and E. A. Lifschits, Some criteria for the existence of periodic oscillation in nonlinear systems, Automat. Remote Control 9 (1973), 1374-1377.

16. W. Krawcewicz, Contribution à la théorie des équations non linéaires dans les espaces de Banach, Dissertationes Math. 273 (1988), 1-83.

17. L. Leray and J. Schauder, Topologie et équations functionnelles, Ann. Sci. École Norm. Sup. 51 (1934), 45-78.

18. J. Mawhin, Periodic solutions of nonlinear functional differential equations, J. Differential Equations 10 (1971), 240-261.

19. __ Equivalence theorems for nonlinear operator equations and coincidence degree theorem for some mappings in locally convex topological vector spaces, J. Differential Equations 12 (1972), 610-636.

20. J. Mawhin, Topological degree methods in nonlinear boundary value problems, CBMS Regional Conf. Ser. in Math., no. 40, Amer. Math. Soc., Providence, R.I., 1977.

21. Compacité monotonie et convexité dans l'étude de problèmes aux limites semi-linéaires, Séminaire d'Analyse Moderne 19, Université de Sherbrooke, 1981.

22. E. M. Muhamadiev and D. B. Sadovskii, Estimation of the spectral radius of a certain operator that is connected with equations of neutral type, Math. Notes 13 (1973), 39-45.

23. R. D. Nussbaum, The fixed point index for local condensing maps, Ann. Mat. Pure Appl. (4) 89 (1971), 217-258.

24. _ The fixed point index and asymptotic fixed point theorems for $k$-set contractions, Bull. Amer. Math. Soc. 75 (1969), 490-495. 
25. __ Degree theory for local condensing maps, J. Math. Anal. Appl. 37 (1972), 741-766.

26. B. N. Sadovskii, Application of topological methods in the theory of periodic solutions of nonlinear differential-operator equations of neutral type, Soviet Math. Dokl. 12 (1971), 15431547.

27. K. Schmidt, Equations différentielles et fonctionnelles non linéaires, Hermann, Paris, 1973, pp. 65-78.

28. P. Volkmann, Démonstration d'un théorème de coincidence par la méthode de Granas, Bull. Soc. Math. Belgique Ser. B 36 (1984), 235-242.

29. P. Waltman and J. S. W. Wong, Two point boundary value problems for nonlinear functional differential equations, Trans. Amer. Math. Soc. 164 (1972), 39-54.

(L. H. Erbe and W. Krawcewicz) Department of Mathematics, University of Alberta, Edmonton, Alberta, Canada T6G 2G1

E-mail address: usererbe@mts.ucs.ualberta.ca

E-mail address: userkraw@mts.ucs.ualberta.ca

(J. H. Wu) Department of Mathematics, York University, North York, Ontario, CANADA M3J 1 P3

E-mail address: wujh@vml.yorku.ca 